\title{
Infusion Related Reaction, CTCAE
}

National Cancer Institute

\section{Source}

National Cancer Institute. Infusion Related Reaction, CT CAE. NCI Thesaurus. Code C143583.

A disorder characterized by adverse reaction to the infusion of pharmacological or biological substances. 